\title{
Interplay between Mesoscopic and Microscopic Fluctuations in Ferromagnets
}

\author{
V.I. Yukalov \\ Centre for Interdisciplinary Studies in Chemical Physics \\ University of Western Ontario, London, Ontario N6A 3K7, Canada \\ and \\ Bogolubov Laboratory of Theoretical Physics \\ Joint Institute for Nuclear Research, Dubna 141980, Russia
}

PACS: 05.20.--y, 05.40.+j, 05.70.Ce, 64.60.-j

keywords: mesoscopic fluctuations, heterophase ferromagnets, phase transitions 


\begin{abstract}
A model of a ferromagnet is considered, in which there arise mesoscopic fluctuations of paramagnetic phase. The presence of these fluctuations diminishes the magnetization of the ferromagnet, softens the spin-wave spectrum, increases the spin-wave attenuation, shortens the magnon free path, lowers the critical point, and can change the order of phase transition. A special attention is paid to the interplay between these mesoscopic paramagnetic fluctuations and microscopic fluctuations due to magnons. One of the main results of this interplay is an essential extension of the region of parameters where the ferromagnet-paramagnet phase transition is of first order.
\end{abstract}




\section{Introduction}

When one introduces into a model of a ferromagnet additional variables, sometimes called hidden, such as phonons or impurities, the behaviour of the model can be essentially distorted. Two main types of changes arise: A renormalization of critical exponents of a continuous transition or the disruption of a continuous transition to the first order one $[1,2]$ (and literature therein). Such changes are caused by the interplay of different fluctuations in a ferromagnet, say, due to magnons and to phonons.

In the present paper we study the interplay between two types of fluctuations in ferromagnets, which has not been examined earlier. Let us consider a ferromagnet in which fluctuations of paramagnetic phase can occur. This kind of fluctuations is called heterophase [3]. Such fluctuations are necessarily of mesoscopic nature, either in space or in time or both. Mesoscopic in space means that their characteristic size is much larger than the average interparticle distance but much smaller than the size of a system. Mesoscopic in time implies that their typical lifetime is much longer than the characteristic time of an elementary oscillation but much shorter than the observation time. An elementary oscillation, in the case of spin systems, concerns fast spin motions whose typical time can be evaluated as $\tau_{l o c} \approx \hbar / J$, where $\hbar$ is the Planck constant and $J$ is an exchange integral. Taking $J \sim\left(10^{-12}-10^{-13}\right)$ erg, we have $\tau_{l o c} \sim\left(10^{-14}-10^{-13}\right) s$. Since heterophase fluctuations are always mesoscopic, we may combine these two notions into one adjective mesophase. Thus, mesophase fluctuations are, by definition, mesoscopic fluctuations of one phase inside a host phase.

Ferromagnets with such mesoscopic fluctuations have been considered earlier [3-5] in the mean-field approximation. The latter takes into account only flippon states [3] of spin systems. However, in real magnets there are also magnon excitations. These excitations describe small oscillations of spins around their equilibrium positions and, thus, are to be treated as microscopic fluctuations.

The aim of this paper is to analyse the properties of a ferromagnet in which there occur both types of fluctuations discussed above, microscopic magnon fluctuations and mesoscopic paramagnetic fluctuations. There are many examples of heterophase magnets (see review [3]) whose mesoscopic heterogeneous structure can be confirmed by neutron scattering experiments, such as diffuse scattering, small-angle scattering, Bragg reflections, and polarized-neutron scattering [6]. This is why it is important to study the properties of mesophase magnets in a more realistic, than mean-field, approximation. Note also that a system with mesophase fluctuations is nothing but another name for a system with mesoscopic phase separation that is now intensively studied in connection with high-temperature superconductors [7-11].

\section{Mesophase Ferromagnet}

Consider a ferromagnet in which there appear mesoscopic paramagnetic fluctuations [12]. Such a system is nonuniform and quasiequilibrium. The general theory of statistical systems with mesoscopic fluctuations is developed in Ref.[3]. Following this theory, 
we average over all possible configurations of heterophase fluctuations coming to an effective Hamiltonian

$$
H_{\text {eff }}=H_{1} \oplus H_{2}
$$

where $H_{\nu}$, with $\nu=1,2$, is a phase-replica Hamiltonian representing a pure ferromagnetic $(\nu=1)$ or paramagnetic $(\nu=2)$ phase. Each $H_{\nu}$ is defined on a weighted space $\mathcal{H}_{\nu}$ of quantum states typical of the corresponding phase [3]. The effective Hamiltonian (1) acts on a yield-weighted fiber space

$$
\mathcal{Y}=\mathcal{H}_{1} \otimes \mathcal{H}_{2}
$$

A phase-replica Hamiltonian $H_{\nu}$, for the Heisenberg-type spin interactions, reads

$$
H_{\nu}=\frac{1}{2} w_{\nu}^{2} N U-w_{\nu}^{2} \sum_{i \neq j} J_{i j} \vec{S}_{i \nu} \cdot \vec{S}_{j \nu}-w_{\nu} \sum_{i} \vec{B} \cdot \vec{S}_{i \nu}
$$

where $N$ is the number of lattice sites; $U$, a crystal-field parameter; $J_{i j}=J_{j i}>0$, an exchange interaction; $\vec{S}_{i \nu}$, a spin operator defined on the weighted space $\mathcal{H}_{\nu}$, so that $\vec{S}_{i \nu} \mathcal{H}_{\nu}=\vec{S}_{i} \mathcal{H}_{\nu} ; i, j=1,2, \ldots, N$

$$
\vec{B}=\mu_{0} H_{0} \vec{e}_{z} \equiv B \vec{e}_{z}
$$

$\mu_{0}$ being a particle magnetic moment; $H_{0}$, external magnetic field in the $z$-direction. The factors $w_{\nu}$ are the geometric phase probabilities satisfying the conditions

$$
0 \leq w_{\nu} \leq 1, \quad w_{1}+w_{2}=1
$$

and defined by minimizing the effective free energy

$$
f=-\frac{T}{N} \ln \operatorname{Tr} \exp \left(-\beta H_{e f f}\right)
$$

in which $T$ is temperature, $\beta T \equiv 1$, and the trace is taken over the fiber space $(2)$. With the notation

$$
w \equiv w_{1}, \quad w_{2} \equiv 1-w,
$$

we have

$$
\frac{\partial f}{\partial w}=\frac{1}{N}\left\langle\frac{\partial}{\partial w} H_{e f f}\right\rangle=0
$$

where $\langle\ldots\rangle$ implies a statistical average with the statistical operator

$$
\hat{\rho}=\frac{\exp \left(-\beta H_{e f f}\right)}{\operatorname{Tr} \exp \left(-\beta H_{e f f}\right)} .
$$

The order parameters

$$
S_{\nu} \equiv \frac{1}{N} \sum_{i=1}^{N}\left\langle S_{i \nu}^{z}\right\rangle \quad(\nu=1,2)
$$


are defined so that for the ferromagnetic phase

$$
\lim _{B \rightarrow 0} \lim _{N \rightarrow \infty} S_{1} \not \equiv 0,
$$

while for the paramagnetic phase

$$
\lim _{B \rightarrow 0} \lim _{N \rightarrow \infty} S_{2} \equiv 0 .
$$

Introducing the notation

$$
\Sigma_{\nu} \equiv \frac{1}{N} \sum_{i \neq j} J_{i j}\left\langle\vec{S}_{i \nu} \cdot \vec{S}_{j \nu}\right\rangle,
$$

we obtain from (7) an equation

$$
w=\frac{U-2 \Sigma_{2}+B\left(S_{1}-S_{2}\right)}{2\left(U-\Sigma_{1}-\Sigma_{2}\right)}
$$

for the ferromagnetic-phase probability. Here it is assumed that $U \neq \Sigma_{1}+\Sigma_{2}$. In the case when the latter assumption does not hold, the equation for $w$ reads as

$$
\Sigma_{1}-\Sigma_{2}+B\left(S_{1}-S_{2}\right)=0 \quad\left(U=\Sigma_{1}+\Sigma_{2}\right) .
$$

In order that (12) would satisfy the probability property $0 \leq w \leq 1$, one of the following sets of inequalities is to be valid, either

$$
\begin{gathered}
U>\Sigma_{1}+\Sigma_{2}, \\
U \geq 2 \Sigma_{1}+B\left(S_{1}-S_{2}\right), \\
U \geq 2 \Sigma_{2}-B\left(S_{1}-S_{2}\right),
\end{gathered}
$$

or

$$
\begin{gathered}
U<\Sigma_{1}+\Sigma_{2}, \\
U \leq 2 \Sigma_{1}+B\left(S_{1}-S_{2}\right), \\
U \leq 2 \Sigma_{2}-B\left(S_{1}-S_{2}\right) .
\end{gathered}
$$

The magnetization of the ferromagnetic phase is expected to be larger than that of the paramagnetic phase, i.e., $S_{1}>S_{2}$. Keeping this in mind, we may notice from (12) that external magnetic field supresses paramagnetic fluctuations, that is, $w_{2} \rightarrow 0$ as $B$ increases. Paramagnetic fluctuations disappear at the critical field

$$
B_{c}=\frac{U-2 \Sigma_{1}}{S_{1}-S_{2}} \quad(w=1),
$$

when $w_{2}=0$. 
Equation (12) for the ferromagnetic probability $w$ resulted from the extremum condition (7). In order that this extremum be a minimum, the inequality

$$
\frac{\partial^{2} f}{\partial w^{2}}>0
$$

must hold. Calculating the second derivative of (6), we take into account (7) and the equality

$$
\frac{\partial \rho}{\partial w}=-\beta \rho \frac{\partial H_{e f f}}{\partial w}
$$

Then we find

$$
\frac{\partial^{2} f}{\partial w^{2}}=\frac{1}{N}\left\langle\frac{\partial^{2} H_{e f f}}{\partial w^{2}}\right\rangle-\frac{\beta}{N}\left\langle\left(\frac{\partial H_{e f f}}{\partial w}\right)^{2}\right\rangle .
$$

The second term in the right-hand side of the latter equality is positively defined (nonnegative). Hence a necessary condition for $\partial^{2} f / \partial w^{2}>0$ is the positiveness of the first term, which gives

$$
U>\Sigma_{1}+\Sigma_{2}
$$

Therefore, between two sets (13) and (14) we must choose the set of inequalities (13). The set (14) can correspond only to a metastable state.

\section{Spin-Wave Approximation}

Consider, first, the low temperatures

$$
T \ll J \equiv \frac{1}{N} \sum_{i \neq j} J_{i j}
$$

when the Dyson $[13,14]$ spin-wave approximation provides accurate asymptotic expansions. Then for the free energy (6) of a mesophase ferromagnet, with spin one half and for zero external field, one has

$$
f \simeq\left(w^{2}-w+\frac{1}{2}\right) U-\frac{w^{2}}{4} J-\frac{T}{w^{3} \rho a_{0}^{3}}\left(\frac{T}{2 \pi J}\right)^{3 / 2}\left[\zeta\left(\frac{5}{2}\right)+\frac{9 T}{8 w^{2} J} \zeta\left(\frac{7}{2}\right)\right],
$$

where $\rho$ is a spin density, $\zeta(\cdot)$ is the Riemann zeta function, and

$$
a_{0}^{2} \equiv \frac{1}{N} \sum_{i \neq j} \vec{r}_{i j}^{2} \frac{J_{i j}}{3 J}, \quad \vec{r}_{i j} \equiv \vec{r}_{i}-\vec{r}_{j}
$$

For the average magnetization

$$
M \equiv \mu_{0}\left(w_{1} S_{1}+w_{2} S_{2}\right)
$$


in the zero field $B=0$, when $S_{2}=0$, we find

$$
\frac{M}{\mu_{0}} \simeq \frac{w}{2}-\frac{\zeta\left(\frac{3}{2}\right)}{w^{2} \rho a_{0}^{3}}\left(\frac{T}{2 \pi J}\right)^{3 / 2},
$$

which shows that, due to disordering caused by mesoscopic fluctuations, the lowtemperature magnetization diminishes.

The spin-wave spectrum [15-17], in the presence of mesophase fluctuations softens, as is seen from the expression

$$
\begin{gathered}
\varepsilon(\vec{k})=2 w^{2} C[J-J(\vec{k})]- \\
-\frac{2 w^{2} C}{(2 \pi)^{3} \rho} \int \frac{J-J(\vec{k})+J(\vec{k}+\vec{q})-J(\vec{q})}{\exp \left(w^{2} J C a_{0}^{2} q^{2} / T\right)-1} d \vec{q}
\end{gathered}
$$

in which

$$
\begin{gathered}
C \equiv S_{1}, \quad J(0)=J, \\
J(\vec{k})=\frac{1}{N} \sum_{i \neq j} J_{i j} e^{-i \vec{k} \vec{r}_{i j}}, \\
J_{i j}=\frac{1}{(2 \pi)^{3} \rho} \int J(\vec{k}) e^{i \vec{k} \vec{r}_{i j}} d \vec{k},
\end{gathered}
$$

and the integration goes over the Brillouin zone [18]. In the long-wave limit, the spin-wave spectrum (19) yields

$$
\varepsilon(\vec{k}) \simeq w^{2} J C a_{0}^{2} k^{2} \quad(k \rightarrow 0) .
$$

The existence of mesophase fluctuations enhances the attenuation $\gamma(k)$ of spin waves due to magnon-magnon scattering and shortens the magnon free path $\lambda(k)$, according to the formulas

$$
\begin{aligned}
& \gamma(k) \simeq \frac{J a_{0}^{2} k^{3}}{2 w^{3}}\left(\frac{2 T}{J}\right)^{5 / 2} \quad(T \ll \varepsilon(\vec{k})), \\
& \lambda(k) \simeq \frac{w^{5} a_{0}^{5} \rho^{2}}{\zeta(3 / 2) k^{2}}\left(\frac{\pi J}{T}\right)^{5 / 2} \quad(T \ll J),
\end{aligned}
$$

which are obtained using the techniques of Refs. [13,14,19]. The shortening of the magnon free path is naturally related to the decrease of the magnon lifetime resulting from the increase of attenuation. 


\section{Mean-Field Approximation}

The model (1)-(3) of a mesophase ferromagnet in the mean-field approximation has been analysed earlier [3-5]. Here we recall only some main formulas and consider the influence of mesophase fluctuations on the phase transition order. The consideration below deals again with spin one half and zero external field. For convenience, we introduce the notation

$$
t \equiv \frac{T}{J}, \quad u \equiv \frac{U}{J} .
$$

For the average spin $C \equiv S_{1}$, we have

$$
C=\frac{1}{2} \tanh \frac{w^{2} C}{t}
$$

The ferromagnetic-phase probability (12) becomes

$$
w=\frac{u}{2\left(u-C^{2}\right)} .
$$

From (22) and (23) we may get

$$
t=u^{2} C\left[2\left(u-C^{2}\right)^{2} \ln \frac{1+2 C}{1-2 C}\right]^{-1} .
$$

In the vicinity of the critical point, when $C \ll 1$, expanding the right-hand side of (24) in powers of $C$, we find

$$
\begin{gathered}
8 t \simeq 1+\left(\frac{2}{u}-\frac{4}{3}\right) C^{2}+\left(\frac{3}{u^{2}}-\frac{8}{u}+2.133\right) C^{4}+ \\
+\left(\frac{4}{u^{3}}-\frac{28}{u^{2}}+\frac{21.333}{u}-3.454\right) C^{6} .
\end{gathered}
$$

This gives either

$$
C \simeq \sqrt{\frac{4 u}{2 u-3}}\left(t_{c}-t\right)^{1 / 2} \quad\left(u \neq \frac{3}{2}\right)
$$

where

$$
t \rightarrow t_{c}-0, \quad t_{c}=\frac{1}{8}
$$

or

$$
C \simeq 1.438\left(t_{c}-t\right)^{1 / 4} \quad\left(u=\frac{3}{2}\right)
$$

depending on the ratio $u \equiv U / J$. The ferromagnet-paramagnet phase transition is of second order for $u>3 / 2$ and of the first order for $u<3 / 2$; the point $u=3 / 2$ being tricritical, where critical indices change their values. 
The order of the phase transition can be checked by using the Landau expansion for the free energy

$$
\frac{f}{J}=\left(w^{2}-w+\frac{1}{2}\right) u+w^{2} C^{2}-t \ln \left[2 \cosh \left(\frac{w^{2} C}{t}\right)\right] .
$$

In our case, we need to substitute here an expansion for the phase probability (23),

$$
w \simeq \frac{1}{2}\left(1+\frac{C^{2}}{u}+\frac{C^{4}}{u^{2}}\right) .
$$

As a result,

$$
\frac{f}{J} \simeq f_{0}+f_{2} C^{2}+f_{4} C^{4},
$$

where the expansion coefficients are

$$
f_{0}=\frac{u}{4}-t \ln 2, \quad f_{2}=-\frac{1-8 t}{32 t}, \quad f_{4}=\frac{1}{4 u}\left(3-\frac{1}{2 t}+\frac{u}{768 t^{3}}\right) .
$$

At the critical point $t_{c}=1 / 8$, we have $f_{2}=0$. The criterion of the second-order transition is $f_{4}>0$ at $t=t_{c}$. Since

$$
f_{4}\left(t_{c}\right)=\frac{2 u-3}{12 u},
$$

we should have either $u<0$ or $u>3 / 2$. The stability conditions (13) and (15) require that $u \geq 2 C^{2}$. Hence, the second-order transition occurs for $u>3 / 2$, while in the interval

$$
0<u<\frac{3}{2}
$$

the transition is of first order. The point $u=3 / 2$ is tricritical. And if $u=0$, we return to the standard case of a pure ferromagnet, without mesophase fluctuations, $w \equiv 1$, with a phase transition at $t_{c}=1 / 2$.

\section{Random-Phase Approximation}

An interesting question, and one of the main for the present paper, is how the inclusion of magnons changes the results of the mean-field consideration for a mesophase ferromagnet, especially in the critical region? To this end, let us introduce the magnon operators [20] for a $\nu$-replica phase by the equalities

$$
b_{j \nu}=S_{j \nu}^{x}+i S_{j \nu}^{y}, \quad b_{j \nu}^{\dagger}=S_{j \nu}^{x}-i S_{j \nu}^{y} .
$$

These operators have the properties

$$
\left[b_{i \nu}, b_{j \nu^{\prime}}\right]=0, \quad b_{i \nu}^{2}=0, \quad\left[b_{i \nu}, b_{j \nu^{\prime}}^{\dagger}\right]=\delta_{i j} \delta_{\nu \nu^{\prime}}\left(1-2 \hat{n}_{i \nu}\right),
$$


where

$$
\hat{n}_{i \nu}=b_{i \nu}^{\dagger} b_{i \nu}
$$

is a magnon density operator in a $\nu$-phase replica. The spin operators are expressed through the magnon operators as

$$
S_{j \nu}^{x}=\frac{1}{2}\left(b_{j \nu}^{\dagger}+b_{j \nu}\right), \quad S_{j \nu}^{y}=\frac{i}{2}\left(b_{j \nu}^{\dagger}-b_{j \nu}\right), \quad S_{j \nu}^{z}=\frac{1}{2}-\hat{n}_{j \nu} .
$$

For a scalar product of two spins, we have

$$
\vec{S}_{i \nu} \cdot \vec{S}_{j \nu}=\frac{1}{4}-\frac{1}{2}\left(\hat{n}_{i \nu}+\hat{n}_{j \nu}\right)+\hat{n}_{i \nu} \hat{n}_{j \nu}+\frac{1}{2}\left(b_{i \nu}^{\dagger} b_{j \nu}+b_{j \nu}^{\dagger} b_{i \nu}\right) .
$$

Define the magnon propagator

$$
G_{i j \nu}(t)=-i\left\langle\hat{T} b_{i \nu}(t) b_{j \nu}^{\dagger}(0)\right\rangle
$$

where $\hat{T}$ is the chronological operator. The random-phase approximation in the evolution equation for propagator (32) implies that

$$
\left\langle b_{i \nu}^{\dagger} b_{i \nu} b_{j \nu}^{\dagger} b_{l \nu}\right\rangle \cong\left\langle b_{i \nu}^{\dagger} b_{i \nu}\right\rangle\left\langle b_{j \nu}^{\dagger} b_{l \nu}\right\rangle .
$$

Employing the Fourier transforms

$$
\begin{gathered}
G_{i j \nu}(t)=\frac{1}{(2 \pi)^{4} \rho} \int G_{\nu}(\vec{k}, \omega) \exp \left\{i\left(\vec{k} \cdot \vec{r}_{i j}-\omega t\right)\right\} d \vec{k} d \omega, \\
G_{\nu}(\vec{k}, w)=\frac{1}{N} \sum_{i j} \int G_{i j \nu}(t) \exp \left\{-i\left(\vec{k} \cdot \vec{r}_{i j}-\omega t\right)\right\} d t
\end{gathered}
$$

we obtain the equation

$$
\left[\omega-\omega_{\nu}(\vec{k})\right] G_{\nu}(\vec{k}, \omega)=2 S_{\nu}
$$

in the random-phase approximation, where the magnon spectrum is

$$
\omega_{\nu}(\vec{k})=2 w_{\nu}^{2} S_{\nu}[J-J(\vec{k})]+w_{\nu} B
$$

The solution of (34) reads

$$
G_{\nu}(\vec{k}, \omega)=2 S_{\nu}\left[\frac{1+n_{\nu}(\vec{k})}{\omega-\omega_{\nu}(\vec{k})+i 0}-\frac{n_{\nu}(\vec{k})}{\omega-\omega_{\nu}(\vec{k})-i 0}\right],
$$

where magnons are treated as bosons and

$$
n_{\nu}(\vec{k})=\frac{1}{\exp \left[\beta \omega_{\nu}(\vec{k})\right]-1}=\frac{1}{2}\left[\operatorname{coth} \frac{\omega_{\nu}(\vec{k})}{2 T}-1\right] .
$$


For an average spin (8) we have

$$
S_{\nu}=\frac{1}{2}-\frac{1}{N} \sum_{j}\left\langle b_{j \nu}^{\dagger} b_{j \nu}\right\rangle .
$$

Using the relation

$$
\begin{gathered}
\left\langle b_{j \nu}^{\dagger} b_{j \nu}\right\rangle=i G_{j j \nu}(-0)=\frac{i}{(2 \pi)^{4} \rho} \int G_{\nu}(\vec{k}, \omega) e^{+i \omega 0} d \vec{k} d \omega= \\
=\frac{2 S_{\nu}}{(2 \pi)^{3} \rho} \int n_{\nu}(\vec{k}) d \vec{k}
\end{gathered}
$$

we obtain the equation

$$
\frac{2 S_{\nu}}{(2 \pi)^{3} \rho} \int \operatorname{coth}\left[\frac{\omega_{\nu}(\vec{k})}{2 T}\right] d \vec{k}=1
$$

for the average spin (38).

For the energy (11), we find

$$
\begin{gathered}
\Sigma_{\nu}=\frac{J}{4}-\frac{1}{2(2 \pi)^{4} \rho} \int\left[J-J(\vec{k})+\frac{\omega}{w_{\nu}^{2}}-\frac{B}{w_{\nu}}\right] e^{-\beta \omega} \times \\
\times I_{\nu}(\vec{k}, \omega) d \vec{k} d \omega,
\end{gathered}
$$

where the spectral function is

$$
I_{\nu}(\vec{k}, \omega) \equiv-\frac{2 \operatorname{Im} G_{\nu}(\vec{k}, \omega)}{1+e^{-\beta \omega}}
$$

Invoking (36), we get

$$
I_{\nu}(\vec{k}, \omega)=\pi S_{\nu} e^{\beta \omega} n_{\nu}(\vec{k}) \delta\left(\omega-\omega_{\nu}(\vec{k})\right)
$$

because of which

$$
\Sigma_{\nu}=\frac{J}{4}-\frac{S_{\nu}\left(1+2 S_{\nu}\right)}{(2 \pi)^{3} \rho} \int[J-J(\vec{k})] n_{\nu}(\vec{k}) d \vec{k} .
$$

The spectral representation (36) for the Green function is written presupposing that magnons are bosons. However, the commutation relations for magnon operators $b_{i \nu}$ demonstrate that magnons are not strictly speaking bosons. Magnons can be approximately treated as bosons provided that the magnon density (the number of magnons per site),

$$
\left\langle\hat{n}_{j \nu}\right\rangle=\left\langle b_{j \nu}^{\dagger} b_{j \nu}\right\rangle=i G_{j j \nu}(-0)
$$


is small, being much smaller that $1 / 2$. This is always correct for a sufficiently strong external field $B$. But if we are interested in the case of zero external field $B=0$, then, according to (9) and (10),

$$
S_{1} \equiv C \not \equiv 0, \quad S_{2} \equiv 0 \quad(B=0) .
$$

And from (38) we get

$$
\left\langle\hat{n}_{i 2}\right\rangle=\frac{1}{2} \quad\left(S_{2}=0\right)
$$

where the translational invariance of the lattice is assumed. Therefore, in zero external field, all formulas, starting from (36) and based on this spectral representation, can be valid only for ferromagnetic phase but not for paramagnetic one.

In zero external field, the magnon spectrum (35) yields

$$
\begin{gathered}
\omega_{1}(\vec{k})=2 w^{2} C[J-J(\vec{k})], \\
\omega_{2}(\vec{k})=0 \quad(B=0) .
\end{gathered}
$$

The soft mode of magnons in paramagnetic phase tells that, in this phase, they are in a kind of condensed state.

The phase probabilities $w_{1} \equiv w$ and $w_{2} \equiv 1-w$ are defined by (12) which, for zero field $B=0$, reads

$$
w=\frac{U-2 \Sigma_{2}}{2\left(U-\Sigma_{1}-\Sigma_{2}\right)} .
$$

To make this equation explicit, we need to find the magnetic energies $\Sigma_{\nu}$ given by (11). For the spin-spin correlation functions, using the random-phase decoupling (33), with $i \neq j$, we have

$$
\begin{gathered}
\left\langle\vec{S}_{i \nu} \cdot \vec{S}_{j \nu}\right\rangle=\left(\left\langle\hat{n}_{i \nu}\right\rangle-\frac{1}{2}\right)\left(\left\langle\hat{n}_{j \nu}\right\rangle-\frac{1}{2}\right)+ \\
+\frac{i}{2}\left[G_{i j \nu}(-0)+G_{j i \nu}(-0)\right] .
\end{gathered}
$$

For ferromagnetic phase, we may employ the spectral representation (36) and get

$$
\left\langle\vec{S}_{i 1} \cdot \vec{S}_{j 1}\right\rangle=C^{2}+\frac{C}{(2 \pi)^{3} \rho} \int \operatorname{coth}\left[\frac{\omega_{1}(\vec{k})}{2 T}\right] \cos \left(\vec{k} \cdot \vec{r}_{i j}\right) d \vec{k},
$$

where $i \neq j$. For paramagnetic phase, (45) gives

$$
\left\langle\vec{S}_{i 2} \cdot \vec{S}_{j 2}\right\rangle=i G_{i j 2}(-0)
$$

To find the Green function for paramagnetic phase, we cannot use (36) but have to return to the equation of motion leading to (34), which results in the equation

$$
\omega G_{2}(\vec{k}, \omega)=0 \quad(B=0) .
$$


The momentum dependence of a Green function comes usually through the spectrum of the corresponding particles. Since the spectrum of paramagnons does not depend on momentum, it is logical to assume that the related Green function also does not depend on it. Then the solution to (48) is of the form

$$
G_{2}(\vec{k}, \omega)=A \delta(\omega)
$$

in which a constant $A$ can be found from conditions (41) and (43). The latter, together with the equality

$$
\frac{1}{(2 \pi)^{3} \rho} \int \exp \left(i \vec{k} \cdot \vec{r}_{i j}\right) d \vec{k}=\delta_{i j}
$$

where the integration goes over the first Brillouin zone, yield $A=-i \pi$. Consequently,

$$
G_{2}(\vec{k}, \omega)=-i \pi \delta(\omega), \quad G_{i j 2}(t)=-\frac{i}{2} \delta_{i j} .
$$

Thus, we see that the correlation function (47) is zero for $i \neq j$, and hence

$$
\Sigma_{2}=\frac{1}{N} \sum_{i \neq j} J_{i j}\left\langle\vec{S}_{i 2} \cdot \vec{S}_{j 2}\right\rangle=0
$$

Now we have all necessary formulas to study thermodynamic characteristics of the ferromagnet with mesoscopic paramagnetic fluctuations. Let us start with low temperatures. Then for the ferromagnon momentum distribution (37), invoking an expansion

$$
\operatorname{coth} x=1+2 \sum_{n=1}^{\infty} e^{-2 n x}
$$

we may write

$$
n_{1}(\vec{k})=\sum_{n=1}^{\infty} \exp \left\{-n \frac{\omega_{1}(\vec{k})}{T}\right\}
$$

For the magnon spectrum, we cam employ an expansion of $J(\vec{k})$ in powers of $\vec{k}$ with the averaging over spherical angles and the use of the equality

$$
\frac{1}{4 \pi} \int \cos ^{n} \theta d \Omega=\frac{1}{n+1} .
$$

This results in

$$
J(\vec{k}) \simeq J\left(1-\frac{1}{2} a_{0}^{2} k^{2}+\frac{1}{40} a_{0}^{4} k^{4}\right), \quad a_{0}^{2} \equiv \frac{1}{3 N} \sum_{i \neq j} \vec{r}_{i j}^{2} \frac{J_{i j}}{J} .
$$

Then, from (44) we find

$$
\omega_{1}(\vec{k}) \simeq J C w^{2} a_{0}^{2} k^{2}\left(1-\frac{a_{0}^{2} k^{2}}{20}\right)
$$


The low-temperature expansion for the average spin becomes

$$
C \simeq \frac{1}{2}-\frac{\zeta(3 / 2)}{w^{3} \rho a_{0}^{3}}\left(\frac{t}{2 \pi}\right)^{3 / 2}-\frac{9 \pi \zeta(5 / 2)}{4 w^{5} \rho a_{0}^{3}}\left(\frac{t}{2 \pi}\right)^{5 / 2},
$$

where notation (21) is used, and $\zeta(\cdot)$ is a Rieman zeta-function. For the magnetic energy, we get

$$
\frac{\Sigma_{1}}{J} \simeq \frac{1}{4}-\frac{3 \pi \zeta(5 / 2)}{w^{5} \rho a_{0}^{3}}\left(\frac{t}{2 \pi}\right)^{5 / 2}-\frac{45 \pi^{2} \zeta(7 / 2)}{4 w^{7} \rho a_{0}^{3}}\left(\frac{t}{2 \pi}\right)^{7 / 2} .
$$

It is evident from both (52) and (53) that the presence of mesophase fluctuations diminishes the average spin and magnetic energy.

The probability of ferromagnetic phase can itself be presented in the form of a low-temperature expansion resulting from (12),

$$
w \simeq w_{0}-\frac{6 \pi \zeta(5 / 2)}{u w_{0}^{3} \rho a_{0}^{3}}\left(\frac{t}{2 \pi}\right)^{5 / 2}-\frac{45 \pi^{2} \zeta(7 / 2)}{2 u w_{0}^{5} \rho a_{0}^{3}}\left(\frac{t}{2 \pi}\right)^{7 / 2},
$$

where $u \equiv U / J$, as in (21), and

$$
w_{0}=\frac{2 u}{4 u-1}
$$

is assumed to be nonzero. The same expansion (54) could be obtained from the equation (7), with the asymptotic form of the free energy

$$
\begin{aligned}
\frac{f}{J} \simeq\left(w^{2}-w\right. & \left.+\frac{1}{2}\right) u-\frac{w^{2}}{4}-\frac{2 \pi \zeta(5 / 2)}{w^{3} \rho a_{0}^{3}}\left(\frac{t}{2 \pi}\right)^{5 / 2}- \\
& -\frac{9 \pi^{2} \zeta(7 / 2)}{2 w^{5} \rho a_{0}^{3}}\left(\frac{t}{2 \pi}\right)^{7 / 2} .
\end{aligned}
$$

Combining (56) and (54), we can find expansions for the specific heat

$$
C_{V} \simeq \frac{15 \zeta(5 / 2)}{4 w^{3} \rho a_{0}^{3}}\left(\frac{t}{2 \pi}\right)^{3 / 2}+\frac{315 \pi \zeta(7 / 2)}{16 w^{5} \rho a_{0}^{3}}\left(\frac{t}{2 \pi}\right)^{5 / 2}
$$

and the entropy

$$
s \simeq \frac{5 \zeta(5 / 2)}{2 w^{3} \rho a_{0}^{3}}\left(\frac{t}{2 \pi}\right)^{3 / 2}+\frac{63 \pi \zeta(7 / 2)}{8 w^{5} \rho a_{0}^{3}}\left(\frac{t}{2 \pi}\right)^{5 / 2} .
$$

The stability condition $\partial^{2} f / \partial w^{2}>0$ for the mesophase system to be stable, at low temperatures, reads

$$
u>\frac{1}{4}+\frac{12 \pi \zeta(5 / 2)}{w^{5} \rho a_{0}^{3}}\left(\frac{t}{2 \pi}\right)^{5 / 2}+\frac{135 \pi^{2} \zeta(7 / 2)}{2 w^{6} \rho a_{0}^{3}}\left(\frac{t}{2 \pi}\right)^{7 / 2} .
$$


It may happen that mesoscopic fluctuations exist only above a temperature $T_{n}$ called the nucleation temperature [3]. The latter can be defined by the condition

$$
w=1, \quad T=T_{n},
$$

leading to an equation $U-2 \Sigma_{1}=0$. If the nucleation temperature lies in the region of low temperatures, then using (53), we obtain

$$
T_{n}=2 \pi J\left[\frac{(1-2 u) \rho a_{0}^{3}}{12 \pi \zeta(5 / 2)}\right]^{2 / 5} .
$$

This formula is valid for $u \leq 1 / 2$.

\section{Phase Transition}

Now pass to the most interesting question, how the interplay between mesoscopic and microscopic fluctuations influences the ferromagnet-paramagnet phase transition? Using the random-phase approximation, we should keep in mind that the latter is not a good approximation for the critical region, as well as the mean-field approximation. Therefore, we do not intend to calculate the critical indices but rather we pay the main attention to the order of the phase transition. Our aim is to understand what qualitative changes occur if one compares two different approximations for a ferromagnet with mesoscopic fluctuations, the mean-field approximation containing no magnon fluctuations and the random-phase approximation including magnon fluctuations.

Consider the critical region, when $C \rightarrow 0$. Then, using an expansion

$$
\operatorname{coth} x \simeq \frac{1}{x}+\frac{x}{3}-\frac{x^{3}}{45} \quad(|x|<\pi),
$$

for the magnon momentum distribution (37), one has

$$
n_{1}(\vec{k}) \simeq \frac{T}{\omega_{1}(\vec{k})}-\frac{1}{2}+\frac{\omega_{1}(\vec{k})}{12 T}-\frac{\omega_{1}^{3}(\vec{k})}{720 T^{3}} .
$$

Introduce the magnon factors

$$
m_{n} \equiv \frac{1}{(2 \pi)^{3} \rho} \int\left[\frac{J-J(\vec{k})}{J}\right]^{n} d \vec{k},
$$

where $n=0, \pm 1, \pm 2, \ldots$ In particular, $m_{0}=1$. Also, assumimg that $J_{i i}=0$, one has $\int J(\vec{k}) d \vec{k}=0$. Therefore, $m_{1}=1$. Denote

$$
m \equiv m_{-1}=\frac{1}{(2 \pi)^{3} \rho} \int \frac{J}{J-J(\vec{k})} d \vec{k}>0 .
$$


The fact that $m>0$ follows from the inequalities

$$
J(\vec{k}) \leq \frac{1}{N}\left|\sum_{i \neq j} J_{i j} \exp \left(-i \vec{k} \cdot \vec{r}_{i j}\right)\right| \leq J
$$

With notation (61), an expansion of (40), as $C \rightarrow 0$, can be written as

$$
\frac{\Sigma_{1}}{J} \simeq \frac{1}{4}-\frac{t}{2 w^{2}}+\frac{1}{2}\left(1-\frac{2 t}{w^{2}}\right) C+\left(1-\frac{m_{2} w^{2}}{6 t}\right) C^{2}-\frac{m_{2} w^{2}}{3 t} C^{3}+\frac{m_{4} w^{6}}{90 t^{3}} C^{4} .
$$

Equation (39) for the average spin yields

$$
3 t\left(2 m t-w^{2}\right)+2 w^{4} C^{2}-\frac{2 m_{3} w^{8}}{15 t^{2}} C^{4} \simeq 0
$$

as $C \rightarrow 0$. And from the equation (12) for the ferromagnetic-phase probability, we find

$$
\begin{gathered}
3 t\left(2 t-w^{2}+4 u w^{2}-2 u w\right)+6 t\left(2 t-w^{2}\right) C+2 w^{2}\left(m_{2} w^{2}-6 t\right) C^{2}+ \\
+4 m_{2} w^{4} C^{3}-\frac{2 m_{4} w^{8}}{15 t^{2}} C^{4} \simeq 0 .
\end{gathered}
$$

For the critical point, defined by the conditions

$$
t=t_{c}, \quad w=w_{c}, \quad C=0,
$$

we obtain

$$
t_{c}=\frac{w_{c}^{2}}{2 m}, \quad w_{c}=\frac{2 m u}{1+m(4 u-1)} .
$$

Introducing the notation

$$
w \equiv w_{c}+\Delta, \quad \tau \equiv \frac{T-T_{c}}{T},
$$

we may rewrite expansions (63) and (64) as follows:

$$
\begin{gathered}
\Delta \simeq \frac{w_{c}}{2 m(4 u-1)}\left\{-\tau+\left[\frac{w_{c}^{2}(-\tau)}{m(4 u-1)}+2(m-1)\right] C+\right. \\
\left.+\left[\frac{2 w_{c}^{2}(m-1)}{m(4 u-1)}+\frac{4 m\left(3-m m_{2} w_{c}^{2}\right)}{3 w_{c}^{2}}+\frac{w_{c}^{5}(-\tau)}{m^{2}(4 u-1)^{2}}-\frac{4 m_{2} w_{c}^{2}(-\tau)}{3(4 u-1)}\right] C^{2}\right\},
\end{gathered}
$$

and

$$
\begin{gathered}
\frac{3 m u(-\tau)}{w_{c}^{3}}+\frac{3}{2 m}\left[m-1+\frac{w_{c}^{2}(-\tau)}{2 m(4 u-1)}\right] C+ \\
+\left[\frac{3}{w_{c}^{2}}-m(4 u-1)-m m_{2}+\frac{3 w_{c}^{2}(m-1)}{2 m^{2}(4 u-1)}+\frac{3(-\tau)}{m(4 u-1)^{2}}-\frac{m_{2} w_{c}^{2}(-\tau)}{m(4 u-1)}\right] C^{2} \simeq 0 .
\end{gathered}
$$


From here, two types of critical behaviour can arise depending on whether the factor $m$ is equal to one or not. In the first case, we get

$$
C \simeq 2\left(\frac{6 u}{4 u-13+m_{2}}\right)^{1 / 2}(-\tau)^{1 / 2} \quad(m=1)
$$

for the average spin, and for $\Delta \equiv w-w_{c}$, we find

$$
\Delta \simeq \frac{32 u\left(12-m_{2}\right)}{4 u-13+m_{2}}(-\tau) \quad(m=1) .
$$

In the case $m \neq 1$, we obtain

$$
C \simeq \frac{2 u m^{2}}{w_{c}^{3}(m-1)} \tau \quad(m \neq 1)
$$

for the order parameter, and also

$$
\Delta \simeq \frac{w_{c}^{3}-4 m^{2} u}{2 m w_{c}^{2}(4 u-1)}(-\tau) \quad(m \neq 1) .
$$

The case $m=1$ leads to formulas (67) and (68) with a mean-field type behaviour, provided that $4 u-13+m_{2}>0$. In order to decide what kind of behaviour would correspond to reality, let us take the values of the parameters $m$ and $m_{2}$ for several crystalline lattices [20]. For the simple cubic lattice, one has $m=1.516$ and $m_{2}=1.167$. For the body-centered cubic lattice, one gets $m=1.393$ and $m_{2}=1.125$. And for the face-centered cubic lattice, one has $m=1.345$ and $m_{2}=1.083$. Stability conditions (13) and (15) require that

$$
u>\frac{m-1}{2 m} \quad\left(t=t_{c}\right)
$$

at the critical point. For a simple cubic lattice this gives $u>0.170$; for b.c.c. lattice, $u>0.141$; and for f.c.c. lattice, $u>0.128$. Since $m \neq 1$, we have to consider formulas (69) and (70). But for $m>1$, the order parameter (69) approaches zero at the critical point (65) from the right side of $t_{c}$. This means that such a critical point, actually, does not exist [3-5], but there is a temperature $t_{0}>t_{c}$ where the first order phase transition occurs.

\section{Conclusion}

We have considered here a model of a ferromagnet with mesoscopic paramagnetic fluctuations. The ferromagnet with such mesophase fluctuations can be thermodynamically more stable than a pure ferromagnet. A necessary condition for the appearance of mesophase fluctuations is a sufficiently strong crystal field.

The occurrence of mesoscopic fluctuations in a ferromagnet leads to a lower, as compared to the case of a pure ferromagnetic phase, magnetization. The spin-wave 
spectrum softens, the spin-wave attenuation increases, and the magnon free path shortens. The laws characterizing these effects can be expressed by the relations

$$
M \sim w, \quad \varepsilon(\vec{k}) \sim w^{2}, \quad \gamma(\vec{k}) \sim w^{-3}, \quad \lambda(\vec{k}) \sim w^{5}
$$

in which $w \leq 1$ is the probability of ferromagnetic phase.

In the mean-field approximation, the order of the ferromagnet-paramagnet phase transition in the mesophase ferromagnet depends on the ratio $u \equiv U / J$ of the crystalfield parameter $U$ to the exchange interaction $J$. For $u \leq 0$, there are no mesophase fluctuations, and the standard second-order transition happens. For $u>0$, mesophase fluctuations exist in some temperature region above the nucleation temperature. In the interval $0<u<3 / 2$, the phase transition becomes of first order. The value $u=3 / 2$ corresponds to a tricritical point. And for $u>3 / 2$, the phase transition in the mesophase ferromagnet is again of second order.

In the random-phase approximation, mesophase fluctuations can exist in the critical region for $u>(m-1) / 2 m$, where the magnon factor $m$ depends on the kind of crystalline lattice, ranging from $m=1.52$ for a simple cubic lattice to $m=1.35$ for a

f.c.c. lattice. At low temperatures, mesophase fluctuations can arise if $u>1 / 4$. The phase transition, for the realistic values of the magnon factor $m>1$, becomes of first order for any $u$ satisfying stability conditions.

\section{Acknowledgement}

I am very grateful for useful remarks to G.A. Gehring, D. ter Haar, H. Hasegawa, R.B. Stinchcombe, and I.R. Yukhnovsky, whom the early variants of this work have been discussed with. The Senior Fellowship from the University of Western Ontario, London, Canada is appreciated. 


\section{References}

[1] M.E. Fisher, Phys. Rev. 176 (1968) 257.

[2] H.W. Capel, L.W.J. Den Ouden, J.H.H. Perk, Physica A 95 (1979) 371.

[3] V.I. Yukalov, Phys. Rep. 208 (1991) 395.

[4] V.I. Yukalov, Physica A 141 (1987) 352.

[5] V.I. Yukalov, Physica A 144 (1987) 369.

[6] Y.A. Alexandrov, Fundamental Properties of Neutron (Clarendon, Oxford, 1992).

[7] V.I. Yukalov, Int. J. Mod. Phys. B 6 (1992) 91.

[8] G. Benedek, K.A. Müller, Eds., Phase Separation in Cuprate Superconductors (World Scientific, Singapore, 1992).

[9] E. Sigmund, K.A. Müller, Eds., Phase Separation in Cuprate Superconductors (Springer, Berlin, 1994).

[10] A.J. Coleman, E.P. Yukalova, V.I. Yukalov, Physica C 243 (1995) 76.

[11] C.T. Shih, Y.C. Chen, T.K. Lee, Phys. Rev. B 57 (1998) 627.

[12] V.I. Yukalov, Oxford Univ. Commun. 47/81, Oxford (1981).

[13] F.J. Dyson, Phys. Rev. 102 (1956) 1217.

[14] F.J. Dyson, Phys. Rev. 102 (1956) 1230.

[15] R. Stinchcombe, G. Horwitz, F. Englert, R. Brout, Phys. Rev. 130 (1963) 155.

[16] V.G. Vaks, A.I. Larkin, S.A. Pikin, J. Exp. Theor. Phys. 26 (1968) 188.

[17] V.G. Vaks, A.I. Larkin, S.A. Pikin, J. Exp. Theor. Phys. 26 (1968) 647.

[18] L. Flax, J. Raich, Phys. Rev. 185 (1969) 797.

[19] A.I. Akhiezer, V.G. Baryakhtar, S.V. Peletminskii, Spin Waves (North Holland, Amsterdam, 1968).

[20] S.V. Tyablikov, Methods in Quantum Theory of Magnetism (Plenum, New York, 1967). 